Finiteness and fluctuations in growing networks

This article has been downloaded from IOPscience. Please scroll down to see the full text article.

2002 J. Phys. A: Math. Gen. 359517

(http://iopscience.iop.org/0305-4470/35/45/302)

View the table of contents for this issue, or go to the journal homepage for more

Download details:

IP Address: 128.197.40.148

The article was downloaded on 06/12/2010 at 20:06

Please note that terms and conditions apply. 


\title{
Finiteness and fluctuations in growing networks
}

\author{
P L Krapivsky and S Redner
}

Center for BioDynamics, Center for Polymer Studies, and Department of Physics, Boston University, Boston, MA 02215, USA

Received 10 July 2002

Published 29 October 2002

Online at stacks.iop.org/JPhysA/35/9517

\begin{abstract}
We study the role of finiteness and fluctuations about average quantities for basic structural properties of growing networks. We first determine the exact degree distribution of finite networks by generating function approaches. The resulting distributions exhibit an unusual finite-size scaling behaviour and they are also sensitive to the initial conditions. We argue that fluctuations in the number of nodes of degree $k$ become Gaussian for fixed degree as the size of the network diverges. We also characterize the fluctuations between different realizations of the network in terms of higher moments of the degree distribution.
\end{abstract}

PACS numbers: $02.50 . \mathrm{Cw}, 05.40 .-\mathrm{a}, 05.50 .+\mathrm{q}$, 87.18.Sn

\section{Introduction}

Networks such as the Internet and the World Wide Web do not grow in an orderly manner. For example, the Web is created by the uncoordinated effort of millions of users and thus lacks an engineered architecture. Although such networks are complex in structure $[1,2]$, their large size is a simplifying feature, and for infinitely large networks the rate equation approach ${ }^{1}$ provides analytical predictions for basic network characteristics. Nevertheless, social and technological networks are not large in a thermodynamic sense (e.g., the number of molecules in a glass of water vastly exceeds the number of routers in the Internet). Thus fluctuations in network properties can be expected to play a more prominent role than in thermodynamic systems $^{2}$. Additionally, extreme properties, such as the degree of the node with the most links in a network $[5,6]$, the website with the most hyperlinks, or the wealth of the richest person in a society are important characteristics of finite systems. The size dependence of these properties or their distribution is difficult to treat within a rate equation approach.

In this paper, we examine the role of finiteness and the nature of fluctuations about mean values for large, but finite growing networks. We shall focus primarily on the degree distribution $N_{k}(N)$, the number of nodes that are linked to $k$ other nodes in a network of $N$ links,

1 A short review of applications of the rate equation approach to network growth is given in [3].

2 It was also suggested that fluctuations can affect the growth of the Web itself (see, e.g., [4]). 
as well as related local structural characteristics. We shall argue that self-averaging holds for the degree distribution, so that the random variables $N_{k}(N)$ become sharply peaked about their average values in the $N \rightarrow \infty$ limit. We shall also argue that the probability distribution for the number of nodes of fixed degree, $P\left(N_{k}, N\right)$, is generally a Gaussian, with fluctuations that vanish as $N \rightarrow \infty$. On the other hand, higher moments of the degree distribution do not self average. This loss of self-averaging ultimately stems from the power-law tail in the degree distribution itself.

In the next section, we define the growing network model and briefly review the behaviour of the average degree distribution in the thermodynamic $N \rightarrow \infty$ limit. We also discuss how the average degree distribution can naturally be expected to attain a finite-size scaling form for large but finite $N$. We then describe our general strategy for studying fluctuations in these growing networks. In section 3 , we outline our simulational approach and present data for the average degree distribution. In the following two sections, we examine the role of finiteness on the degree distribution, both within a continuous formulation based on the rate equations (section 4), and an exact discrete approach (section 5). The former approach is the one that is conventionally applied to study the kinetics of evolving systems, such as growing networks. While this approach has the advantage of simplicity and it provides an accurate description for the degree distribution in an appropriate degree range, it is quantitatively inaccurate in the large degree limit. This is the domain where discreteness effects play an important role and the exact discrete recursion relations for the evolution of the degree distribution are needed to fully account its properties. In section 6 , we discuss the implications of our results for higher moments of the degree distribution and their associated fluctuations. Section 7 provides conclusions and some perspectives. Calculational details are given in the appendices.

\section{Statement of the problem}

The growing networks considered in this work are built by adding nodes to the network one at a time according to the rule that each new node attaches to a single previous node with a rate proportional to $A_{k}$, where $k$ is the degree of the target node. We investigate the class of models in which $A_{k}=k+\lambda$, where $\lambda>-1$, but is otherwise arbitrary. The general situation of $-1<\lambda<\infty$ corresponds to linear preferential attachment, but with an additive shift $\lambda$ in the rate. This model was originally introduced by Simon to account for the word frequency distribution [7]. The case $\lambda=0$ corresponds to the Barabási-Albert model [8], while the limit $\lambda \rightarrow \infty$ corresponds to random attachment in which each node has an equal probability of attracting a connection from the new node. Thus by varying $\lambda$, we can tune the relative importance of popularity in the attachment rate.

Previous work on the structure of such networks was primarily concerned with the configuration-averaged degree distribution $\left\langle N_{k}(N)\right\rangle$, where the angle brackets denote an average over all realizations of the growth process for an ensemble of networks with the same initial condition. Additionally, most studies focused on the tail region where $k$ is much smaller than any other scale in the system. For attachment rate $A_{k}=k+\lambda$, this average degree distribution has a power-law tail [7, 9],

$$
\left\langle N_{k}(N)\right\rangle=N n_{k} \quad \text { with } \quad n_{k} \propto k^{-(3+\lambda)}
$$

as $N \rightarrow \infty$. In the specific case of $A_{k}=k$, the average degree distribution explicitly is [7-11]

$$
\left\langle N_{k}(N)\right\rangle=N n_{k} \quad \text { with } \quad n_{k}=\frac{4}{k(k+1)(k+2)} .
$$

For finite $N$, however, the degree distribution must eventually deviate from these predictions because the maximal degree cannot exceed $N$. To establish the range of applicability 
of equation (1), we estimate the magnitude of the largest degree in the network, $k_{\max }$ by the extreme statistics criterion $\sum_{k \geqslant k_{\max }}\left\langle N_{k}(N)\right\rangle \approx 1$. This yields $k_{\max } \propto N^{1 /(2+\lambda)}$. We, therefore, anticipate that the average degree distribution will deviate from equation (1) when $k$ becomes of the order of $k_{\max }$. The existence of a maximal degree also suggests that the average degree distribution should attain a finite-size scaling form

$$
\left\langle N_{k}(N)\right\rangle \simeq N n_{k} F(\xi) \quad \xi=k / k_{\max } .
$$

Some aspects of these finite-size corrections were recently studied in [12-15]. One basic result of our work is that we can compute the scaling function explicitly. We find that this function is peaked for $k$ of the order of $k_{\max }$ and that it depends substantially on the initial condition. In contrast, the small-degree tail of the distribution-the reason why such networks were dubbed scale-free-is independent of $N$ and the initial condition.

To study finite networks where fluctuations can be significant, we need a stochastic approach rather than a deterministic rate equation formulation. For finite $N$, the state of a network is generally characterized by the set $\mathbf{N}=\left\{N_{1}, N_{2}, \ldots\right\}$ that occurs with probability $P(\mathbf{N})$. The network state $\mathbf{N}$ evolves by the following processes:

$$
\left(N_{1}, N_{2}\right) \rightarrow\left(N_{1}, N_{2}+1\right) \quad\left(N_{1}, N_{k}, N_{k+1}\right) \rightarrow\left(N_{1}+1, N_{k}-1, N_{k+1}+1\right) .
$$

The first process corresponds to the new node attaching to an existing node of degree 1 ; in this case, the number of nodes of degree 1 does not change while the number of nodes of degree 2 increases by 1 . The second line accounts for the new node attaching to a node of degree $k>1$.

From these processes, it is straightforward, in principle, to write the master equation for the joint probability distribution $P(\mathbf{N})$. It turns out that correlation functions of a given order are coupled only to correlation functions of the same and lower orders. Thus, we do not need to invoke factorization (as in the kinetic theory) and we could, in principle, solve for correlation functions recursively. However, this would provide much more information than is of practical interest. Typically, we are interested in the degree distribution, or perhaps twobody correlation functions of the form $\left\langle N_{i} N_{j}\right\rangle$. Even though straightforward in principle, it is difficult to compute even the two-point correlation functions $\left\langle N_{i} N_{j}\right\rangle$ for general $i$ and $j$. In this work, we shall restrict ourselves to the specific (and simpler) examples of $\left\langle N_{1}^{2}\right\rangle,\left\langle N_{1} N_{2}\right\rangle$ and $\left\langle N_{2}^{2}\right\rangle$. We will use these results to help characterize fluctuations in finite networks.

\section{Simulation method and data}

To simulate a network with attachment rate $A_{k}=k+\lambda$ efficiently, we exploit an equivalence to the growing network with re-direction (GNR) [9]. In the GNR, a newly-introduced node $\mathbf{n}$ selects an earlier 'target' node $\mathbf{x}$ uniformly. With probability $1-r$, a link from $\mathbf{n}$ to $\mathbf{x}$ is created. However, with probability $r$, the link is re-directed to the ancestor node $\mathbf{y}$ of node $\mathbf{x}$ (figure 1). As discussed in [9], the GNR is equivalent to a growing network with the attachment rate $A_{k}=k+\lambda$, with $\lambda=r^{-1}-2$. Thus, for example, the GNR with $r=1 / 2$ corresponds to the growing network with linear preferential attachment, $A_{k}=k$. Simulation of the GNR is extremely simple because the selection of the initial target node is purely random and the ensuing re-direction step is local.

There is, however, an important subtlety about this equivalence that was not discussed previously in [9]. Namely, the redirection process does not apply when a node has no ancestor. By construction, every node that is added to the network does have a single ancestor, but some 


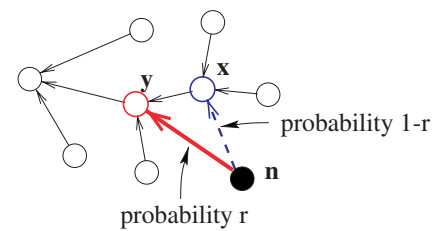

Figure 1. The re-direction process. The new node $\mathbf{n}$ selects a random target node $\mathbf{x}$. With probability $1-r$ a link is established to this target node (dashed), while with probability $r$ the link is established to $\mathbf{y}$, the ancestor of $\mathbf{x}$ (solid).

primordial nodes may have none. For example, for the very natural 'dimer' initial condition $\circ \longleftarrow$, the seed node on the left has no ancestor and the GNR construction for this node is ambiguous. One way to resolve this dilemma is to adopt the 'triangle' initial condition in which there are three nodes in a triangle with cyclic connections between nodes. This leads to the correct attachment rate for each node for any value of $\lambda$. We therefore typically use this initial state to generate degree distribution data. On the other hand, theoretical analysis is simpler for the dimer initial condition. This state can also be simulated in a simple manner (for the case $\lambda=0$ ) by a slightly modified GNR construction in which direct attachment to the seed node is not allowed. It is straightforward to check that this additional rule leads to the correct attachment rates for all the nodes in the network.

Figure 2 shows the average degree distribution for attachment rates $A_{k}=k$ and $A_{k}=k+\lambda$ with $\lambda=-0.9$ for the triangle initial condition. This latter value of $\lambda$ gives results that are representative for values of $\lambda$ close to -1 . The data exhibit a shoulder at $k \approx k_{\max }$ that is much more pronounced when $\lambda<0$ (figure $2(b)$ ). This shoulder is also at odds with the natural expectation that the average degree distribution should exhibit a monotonic cutoff when $k$ becomes of the order of $k_{\max }$. This shoulder turns into a clearly-resolved peak that exhibits relatively good data collapse when the degree distribution is re-expressed in the scaling form of equation (3) (figure 3). Conversely, the magnitude of the peak diminishes rapidly when $\lambda$ is positive and becomes imperceptible for $\lambda \gtrsim 0.5$.

In the following two sections, we will attempt to understand this anomalous feature of the degree distribution by studying the rate equations for the node degrees of finite networks.

\section{Continuum formulation}

We focus on the case of the linear attachment rate $A_{k}=k$ and briefly quote corresponding results for other attachment rates. In the continuum approach, $N$ is treated as continuously varying. Then the change in the average degree distribution satisfies the rate equation

$$
\frac{\mathrm{d}\left\langle N_{k}(N)\right\rangle}{\mathrm{d} N}=\left\langle\frac{(k-1) N_{k-1}(N)-k N_{k}(N)}{2 N}\right\rangle+\delta_{k, 1} .
$$

We assume the dimer initial state - two nodes connected by a single link so that $N_{k}(N=1)=$ $2 \delta_{k, 1}$.

Equations (4) are recursive and can be solved sequentially, starting with $\left\langle N_{1}\right\rangle$. Explicit results for $\left\langle N_{k}\right\rangle, k \leqslant 4$, are given in appendix A. These expressions show that the dominant contribution in the $N \rightarrow \infty$ limit is linear in $N$ and this corresponds to the solution in equation (2). Indeed, if we substitute $\left\langle N_{k}(N)\right\rangle=n_{k} N$ into equations (4), we obtain the recursion $n_{k}=n_{k-1}(k-1) /(k+2)$, whose solution is equation (2). From the first few $\left\langle N_{k}\right\rangle$, 

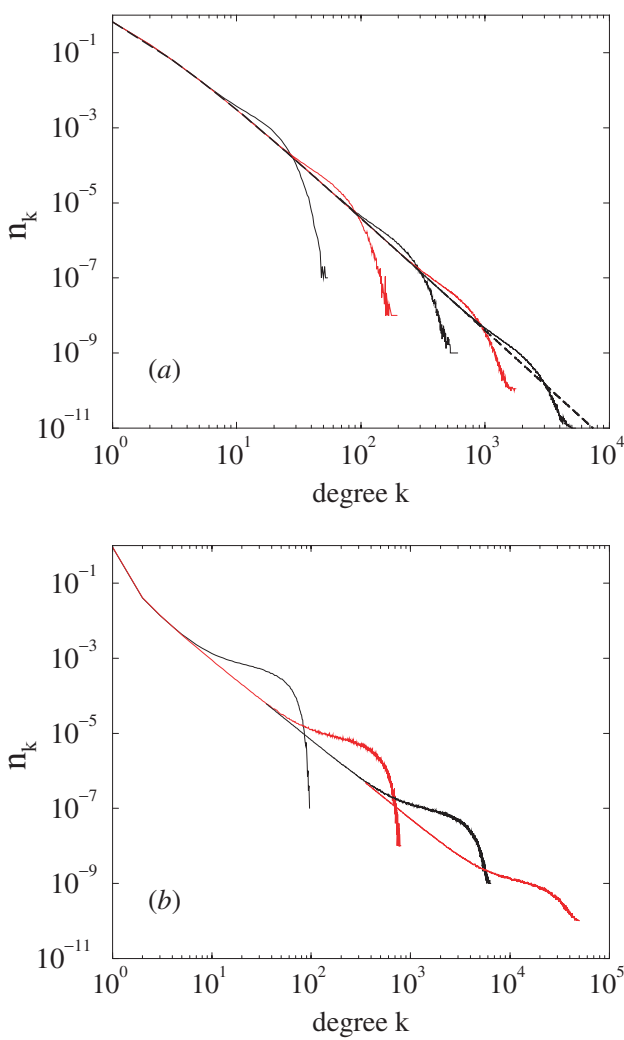

Figure 2. Normalized degree distributions for the triangle initial condition for networks of $10^{2}, 10^{3}, \ldots$ links (upper left to lower right), with $10^{5}$ realizations for each $N$, for $(a) A_{k}=k$ (up to $10^{6}$ links) and $(b) A_{k}=k+\lambda$, with $\lambda=-0.9$ (up to $10^{5}$ links). In $(a$ ), the dashed line is the asymptotic result $n_{k}=4 /[k(k+1)(k+2)]$; the last three data sets were averaged over 3,9 and 27 points, respectively. In $(b)$, the last two data sets were averaged over 10 and 100 points, respectively.

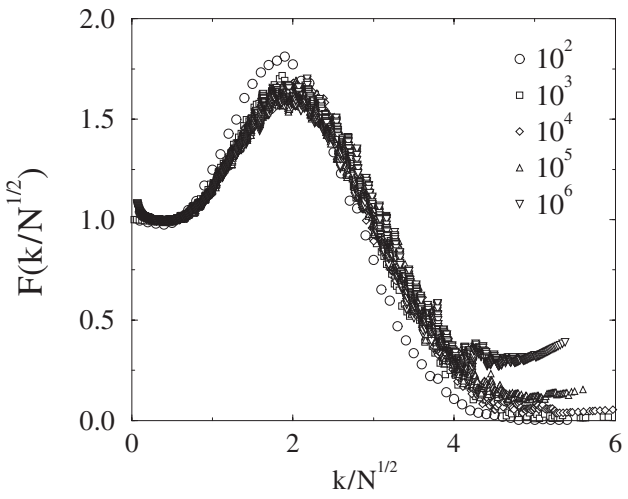

Figure 3. The corresponding scaling function $F(\xi)$ in equation (3) for the data in figure $2(a)$.

it is easy to see that the first correction to this leading behaviour is of the order of $N^{-1 / 2}$. Substituting $\left\langle N_{k}(N)\right\rangle=n_{k} N+A_{k} N^{-1 / 2}$ into equations (4) and keeping the first two terms 
in each $\left\langle N_{k}\right\rangle$, we find $A_{k}=4 / 3$. Continuing this procedure systematically, we arrive at the expansion

$$
\begin{array}{rl}
\left\langle N_{k}(N)\right\rangle=n_{k} & N+\frac{4}{3} \frac{1}{N^{1 / 2}}-\frac{3}{2} \frac{k-1}{N}+\frac{4}{5} \frac{(k-1)(k-2)}{N^{3 / 2}} \\
& -\frac{5}{18} \frac{(k-1)(k-2)(k-3)}{N^{2}}+\frac{1}{14} \frac{(k-1)(k-2)(k-3)(k-4)}{N^{5 / 2}}+\cdots
\end{array}
$$

In general, the right-hand side contains $k+1$ terms which can be written more succinctly as

$$
\left\langle N_{k}(N)\right\rangle=n_{k} N+\frac{1}{N^{1 / 2}} \sum_{j=0}^{k-1} \frac{\Gamma(k)}{\Gamma(k-j)} \frac{(-1)^{j} v_{j}}{N^{j / 2}} .
$$

The coefficients $v_{j}=(2 j+4) /[j !(j+3)]$ may be obtained by imposing the initial condition $N_{k}(1)=2 \delta_{k, 1}$ as each $\left\langle N_{k}\right\rangle$ is computed; a simpler way of obtaining these coefficients will be explained below. Note that expansion (5) is asymptotic because successive terms decrease only when $k \ll \sqrt{N}$.

A more convenient way to solve equations (4) is in terms of the generating function

$$
\mathcal{N}(N, z)=\sum_{k=1}^{\infty}\left\langle N_{k}(N)\right\rangle z^{k}
$$

Multiplying equation (4) by $z^{k}$ and summing over $k$, the generating function satisfies the following partial differential equation:

$$
\left(2 N \frac{\partial}{\partial N}+z(1-z) \frac{\partial}{\partial z}\right) \mathcal{N}(N, z)=2 N z .
$$

The initial condition is $\mathcal{N}(1, z)=2 z$, corresponding to a starting point of two nodes and a single connecting link.

We reduce equation (8) to a wave equation with constant coefficients by changing from the variables $(N, z)$ to $(\ln \sqrt{N}, \ln [z /(1-z)])$. Then by introducing the rotated coordinates $x, y$ such that $x+y=\ln \sqrt{N}$ and $x-y=\ln [z /(1-z)]$, we recast the wave equation into

$$
\frac{\partial \mathcal{N}(x, y)}{\partial x}=\frac{2 \mathrm{e}^{3 x+2 y}}{\mathrm{e}^{x}+\mathrm{e}^{y}}
$$

whose general solution is

$$
\mathcal{N}(x, y)=\mathrm{e}^{2 x+2 y}-2 \mathrm{e}^{x+3 y}+2 \mathrm{e}^{4 y} \ln \left(\mathrm{e}^{x}+\mathrm{e}^{y}\right)+G(y) .
$$

Finally, $G(y)$ is found by imposing the initial condition $\mathcal{N}(1, z)=2 z$. When $N=1$, we have $x=-y$, so that the initial condition becomes $\mathcal{N}(-y, y)=2 /\left(1+\mathrm{e}^{2 y}\right)$. Therefore

$$
G(y)=\frac{2}{1+\mathrm{e}^{2 y}}-1+2 \mathrm{e}^{2 y}-2 \mathrm{e}^{4 y} \ln \left(\mathrm{e}^{-y}+\mathrm{e}^{y}\right)
$$

and finally

$$
\mathcal{N}(x, y)=\mathrm{e}^{2 y}\left(\mathrm{e}^{2 x}-2 \mathrm{e}^{x+y}+2\right)+\frac{1-\mathrm{e}^{2 y}}{1+\mathrm{e}^{2 y}}+2 \mathrm{e}^{4 y} \ln \left(\frac{\mathrm{e}^{x+y}+\mathrm{e}^{2 y}}{1+\mathrm{e}^{2 y}}\right) .
$$

Using $\mathrm{e}^{2 y}=\left(z^{-1}-1\right) \sqrt{N}$ and $\mathrm{e}^{x+y}=\sqrt{N}$, we re-express the generating function in terms of the original variables

$$
\begin{aligned}
\mathcal{N}(N, z)=(3 & \left.-2 z^{-1}\right) N+2\left(z^{-1}-1\right) \sqrt{N}+\frac{1-\left(z^{-1}-1\right) \sqrt{N}}{1+\left(z^{-1}-1\right) \sqrt{N}} \\
& -2\left(z^{-1}-1\right)^{2} N \ln \left(1-z+\frac{z}{\sqrt{N}}\right) .
\end{aligned}
$$


We are primarily interested in the degree distribution for nodes whose degree is of the order of $k_{\max } \approx \sqrt{N}$. This part of the distribution can be extracted from the limiting behaviour of the generating function $\mathcal{N}(N, z)$ as $z \rightarrow 1$ from below. Since the interesting range is $k \approx \sqrt{N}$, it is convenient to write

$$
z^{-1}=1+\frac{s}{\sqrt{N}}
$$

and keep $s$ finite while taking $N \rightarrow \infty$ limit. We simplify still further by eliminating the contribution to the generating function from the power-law tail of $n_{k}$ in equation (2). For this purpose we consider the modified generating function

$$
\left(z^{2} \frac{\partial}{\partial z}\right)^{3} \mathcal{N}=\sum_{k=1}^{\infty}(k+2)(k+1) k\left\langle N_{k}\right\rangle z^{k+3}
$$

which is constructed so that the derivatives multiply the degree distribution by just the right factors to eliminate the power-law tail. The leading behaviour of this modified generating function will therefore provide the scaling function $F(\xi)$ of equation (3).

We now substitute equation (12) and the anticipated scaling form of equation (3) into the right-hand side of equation (13) and replace the sum by an integral. This gives the Laplace transform of the scaling function times a prefactor,

$$
4 N^{3 / 2} \int_{0}^{\infty} \mathrm{d} \xi F(\xi) \mathrm{e}^{-\xi s}
$$

with $\xi=k / N^{1 / 2}$. Using equation (11), we compute the derivative on the left-hand side of equation (13). In the $N \rightarrow \infty$ limit, this derivative becomes $4 N^{3 / 2} J(s)$ with

$$
J(s)=\frac{1}{1+s}+\frac{1}{(1+s)^{2}}+\frac{1}{(1+s)^{3}}+\frac{3}{(1+s)^{4}} .
$$

This is just the Laplace transform of the scaling function. Inverting the Laplace transform then yields

$$
F(\xi)=(1+\xi)\left(1+\frac{\xi^{2}}{2}\right) \mathrm{e}^{-\xi}
$$

Note that the coefficients $v_{j}$ in equation (6) can be obtained by expanding $F$ in a Taylor series. This is a much simpler approach than solving each $\left\langle N_{k}(N)\right\rangle$ directly.

An important feature of the degree distribution is that it depends significantly on the initial condition. For example, for the triangle initial condition, solving equation (8) subject to $N_{k}^{\Delta}(N=3)=3 \delta_{k, 2}$, or $\mathcal{N}^{\Delta}(3, z)=3 z^{2}$, yields

$$
\begin{aligned}
\mathcal{N}^{\Delta}(N, z)= & \left(3-2 z^{-1}\right) N+2\left(z^{-1}-1\right) \sqrt{3 N}+3\left(1+\left(z^{-1}-1\right) \sqrt{N / 3}\right)^{-2}-3 \\
& -2\left(z^{-1}-1\right)^{2} N \ln \left(1-z+z \sqrt{\frac{3}{N}}\right) .
\end{aligned}
$$

Repeating the steps used to deduce the scaling function (16) from equation (11), we now find

$$
F^{\Delta}(\xi)=\left(1+\eta+\frac{\eta^{2}}{2}+\frac{\eta^{4}}{4}\right) \mathrm{e}^{-\eta} \quad \eta \equiv \xi \sqrt{3}
$$

Therefore, small differences in the initial condition translate to discrepancies of the order of $\sqrt{N}$ in the degree distribution of a finite network of $N$ links. Thus, the properties of the nodes with the largest degrees are quite sensitive to the first few growth steps of the network (see also [6]). 
While this initial condition dependence is real, there is also a spurious aspect to this effect. This may be illustrated by considering the linear trimer initial condition $\circ-\circ-\circ$. This is the unique outcome of the dimer initial condition after one node has been added. These two initial conditions should therefore lead to the same degree distribution. However, for the linear trimer initial state $\left(N_{k}(N=2)=2 \delta_{k, 1}+\delta_{k, 2}\right)$ the continuum approach gives the scaling function

$$
F(\xi)=\left(1+\eta+\frac{\eta^{2}}{2}+\frac{\eta^{3}}{4}+\frac{\eta^{4}}{8}\right) \mathrm{e}^{-\eta} \quad \eta \equiv \xi \sqrt{2}
$$

which is distinct from equation (16)! This anomaly highlights one basic limitation of the continuum formulation.

Finally, we mention that parallel results can be obtained for the general case of the shifted linear attachment rate, $A_{k}=k+\lambda$. The rate equation for the average degree distribution is

$$
\frac{\mathrm{d}\left\langle N_{k}(N)\right\rangle}{\mathrm{d} N}=\left\langle\frac{A_{k-1} N_{k-1}(N)-A_{k} N_{k}(N)}{A}\right\rangle+\delta_{k, 1}
$$

where $A=\sum A_{k} N_{k}=\sum(k+\lambda) N_{k}$. To compute $A$ we use the sum rules $\sum k N_{k}=2 N$ (every link contributes twice to the total degree), as well as $\sum N_{k}=N+1$ (for any three initial condition) or $\sum N_{k}=N$ (for an initial condition that has the topology of a single cycle). To simplify final formulae, we use the latter topology (specifically, the triangle initial condition) so that $A=(2+\lambda) N$.

Solving the above rate equations successively, we find that the first two terms in the asymptotic series for $\left\langle N_{k}^{\Delta}(N)\right\rangle$ are

$$
\left\langle N_{k}^{\Delta}(N)\right\rangle \sim n_{k} N+n_{k}^{\prime} N^{-(1+\lambda) /(2+\lambda)}
$$

with

$$
\begin{aligned}
& n_{k}=(2+\lambda) \frac{\Gamma(3+2 \lambda)}{\Gamma(1+\lambda)} \frac{\Gamma(k+\lambda)}{\Gamma(k+3+2 \lambda)} \\
& n_{k}^{\prime}=-\frac{2+\lambda}{3+2 \lambda} \frac{3^{(3+2 \lambda) /(2+\lambda)}}{\Gamma(1+\lambda)} \frac{\Gamma(k+\lambda)}{\Gamma(k)} .
\end{aligned}
$$

The corresponding leading behaviours are $n_{k} \propto k^{-(3+\lambda)}$ and $n_{k}^{\prime} \propto k^{\lambda}$. Thus the two contributions to the degree distribution in equation (19) are comparable when $k \approx N^{1 /(2+\lambda)}$. This value coincides with the maximal degree $k_{\max }$ that is obtained by the extreme value condition $\sum_{k \geqslant k_{\max }} N / k^{3+\lambda} \approx 1$. Once again, the degree distribution is described by a scaling function in the dimensionless variable $\xi=k / N^{1 /(2+\lambda)}$.

\section{Discrete approach}

We now turn to the discrete approach for the network evolution. That is, one link is introduced at each discrete time step; this corresponds exactly to what occurs in the simulation. We again focus on the case of the linear attachment rate $A_{k}=k$. We first treat in detail the case of nodes of degree one and then extend our approach to nodes of higher degrees. Finally, we give a scaling description for the degree distribution itself.

\subsection{Nodes of degree one}

The number of nodes of degree one, $N_{1}(N)$, is a random variable that changes according to

$$
N_{1}(N+1)= \begin{cases}N_{1}(N) & \text { prob. } \frac{N_{1}}{2 N} \\ N_{1}(N)+1 & \text { prob. } 1-\frac{N_{1}}{2 N}\end{cases}
$$


after each node addition event. That is, with probability $N_{1} / 2 N$, a newly-introduced node attaches to a node of degree one; in this case, the number of nodes of degree one does not change. Conversely, with probability $\left(1-N_{1} / 2 N\right)$, the new node attaches to a node of degree greater than one and $N_{1}$ thus increases by one. Therefore

$$
\left\langle N_{1}(N+1)\right\rangle=\left\langle\frac{N_{1}^{2}(N)}{2 N}\right\rangle+\left\langle N_{1}(N)+1-\frac{N_{1}^{2}(N)}{2 N}-\frac{N_{1}(N)}{2 N}\right\rangle
$$

from which

$$
\left\langle N_{1}(N+1)\right\rangle=1+\left(1-\frac{1}{2 N}\right)\left\langle N_{1}(N)\right\rangle
$$

We take the initial condition $\left\langle N_{1}(1)\right\rangle=N_{1}(1)=2$.

We solve this recursion in terms of the generating function $\mathcal{X}_{1}(w)=\sum_{N \geqslant 1}\left\langle N_{1}(N)\right\rangle w^{N-1}$. We, therefore, multiply equation (21) by $N w^{N-1}$ and sum over $N \geqslant 1$ to convert this recursion into the differential equation

$$
\frac{\mathrm{d} \mathcal{X}_{1}}{\mathrm{~d} w}=\frac{1}{(1-w)^{2}}+\frac{1}{2} \mathcal{X}_{1}+w \frac{\mathrm{d} \mathcal{X}_{1}}{\mathrm{~d} w}
$$

Solving equation (22) subject to the initial condition $\mathcal{X}_{1}(0)=2$ gives

$$
\mathcal{X}_{1}(w)=\frac{2}{3} \frac{1}{(1-w)^{2}}+\frac{4}{3} \frac{1}{(1-w)^{1 / 2}} .
$$

Finally, we expand $\mathcal{X}_{1}(w)$ in a Taylor series in $w$ to obtain

$$
\left\langle N_{1}(N)\right\rangle=\frac{2}{3} N+\frac{4}{3 \sqrt{\pi}} \frac{\Gamma\left(N-\frac{1}{2}\right)}{\Gamma(N)} .
$$

The leading term is identical to that in the continuum approach (cf appendix A), but the coefficient of the correction term is $4 /(3 \sqrt{\pi}) \approx 0.7523$, compared to $4 / 3$ in the continuum approach.

The discrete approach is also suited to analysing higher moments of the random variable $N_{1}(N)$. The second moment $\left\langle N_{1}^{2}(N)\right\rangle$ plays an especially important role as we can then obtain the variance $\sigma_{1}^{2}=\left\langle N_{1}^{2}(N)\right\rangle-\left\langle N_{1}(N)\right\rangle^{2}$ and thereby quantify fluctuations. From equation (20) this second moment $\left\langle N_{1}^{2}(N)\right\rangle$ obeys the following recursion formula:

$$
\left\langle N_{1}^{2}(N+1)\right\rangle=1+\left(1-\frac{1}{N}\right)\left\langle N_{1}^{2}(N)\right\rangle+\left(2-\frac{1}{2 N}\right)\left\langle N_{1}(N)\right\rangle .
$$

The solution to this recursion is outlined in appendix B and the final result is

$$
\left\langle N_{1}^{2}(N)\right\rangle=\frac{4}{9} N(N+1)-\frac{1}{3} N+\frac{16}{9 \sqrt{\pi}} \frac{\Gamma\left(N+\frac{1}{2}\right)}{\Gamma(N)}-\frac{4}{3 \sqrt{\pi}} \frac{\Gamma\left(N-\frac{1}{2}\right)}{\Gamma(N)}+\frac{35}{9} \delta_{N, 1} .
$$

In the large $N$ limit, we use Stirling's formula to give, for the variance,

$$
\sigma_{1}^{2}=\frac{N}{9}-\frac{20}{9 \sqrt{\pi}} \frac{1}{N^{1 / 2}}-\frac{16}{9 \sqrt{\pi}} \frac{1}{N}+\cdots .
$$

To obtain the entire probability distribution $P\left(N_{1}, N\right)$ one must solve

$$
P\left(N_{1}, N+1\right)=\frac{N_{1}}{2 N} P\left(N_{1}, N\right)+\left(1-\frac{N_{1}-1}{2 N}\right) P\left(N_{1}-1, N\right) .
$$

By the Markov nature of the process, $P\left(N_{1}, N\right)$ should approach a Gaussian distribution in the large $N$ limit. Numerically, we indeed find a Gaussian distribution with a peak at $2 N / 3$ and dispersion $\frac{1}{3} \sqrt{N}$ in agreement with our theoretical results for $\left\langle N_{1}(N)\right\rangle$ and $\left\langle N_{1}^{2}(N)\right\rangle$. 


\subsection{Degree greater than one}

For $k \geqslant 2$, the random variable $N_{k} \equiv N_{k}(N)$ changes according to

$$
N_{k}(N+1)= \begin{cases}N_{k}-1 & \text { prob. } \frac{k N_{k}}{2 N} \\ N_{k}+1 & \text { prob. } \frac{(k-1) N_{k-1}}{2 N} \\ N_{k} & \text { prob. } 1-\frac{(k-1) N_{k-1}+k N_{k}}{2 N}\end{cases}
$$

at each node addition event. Again, because of the Markov nature of this process, we anticipate that $P\left(N_{k}, N\right)$ approaches a Gaussian distribution for every fixed degree $k$; therefore, we only need to calculate $\left\langle N_{k}(N)\right\rangle$ and $\left\langle N_{k}^{2}(N)\right\rangle$ to infer the asymptotic distribution. To determine the first moment, we repeat the steps described in detail for $k=1$ and obtain the recursion formula

$$
\left\langle N_{k}(N+1)\right\rangle=\left\langle N_{k}(N)\right\rangle+\left\langle\frac{(k-1) N_{k-1}(N)-k N_{k}(N)}{2 N}\right\rangle .
$$

The solution to this recursion is given in appendix $\mathrm{C}$ and explicit formulae for $\left\langle N_{k}(N)\right\rangle$ for $k \leqslant 5$ are also quoted. Qualitatively, these results closely correspond to the asymptotic series for $\left\langle N_{k}(N)\right\rangle$ in the continuum formulation (equation (5)) but with somewhat different coefficients in the correction terms.

The determination of the second moment $\left\langle N_{k}^{2}\right\rangle$ is more complicated because it is coupled to $\left\langle N_{k-1} N_{k}\right\rangle$, which in turn is coupled to $\left\langle N_{k-2} N_{k}\right\rangle$, etc. However, we can still determine $\left\langle N_{k}^{2}\right\rangle$ for small $k$ (appendix D). From the structure of the rate equations, our general conclusion is that $\sigma_{k}^{2}=\left\langle N_{k}^{2}(N)\right\rangle-\left\langle N_{k}(N)\right\rangle^{2}=\mu_{k} N$. Therefore the distribution of $N_{k}(N)$ approaches a Gaussian for each $k$ as $N \rightarrow \infty$.

\subsection{Generating function approach}

In close analogy with section 3, we now obtain the generating function for $\left\langle N_{k}(N)\right\rangle$, from which the exact scaling function in equation (3) can be deduced. Since equation (30) involves two discrete variables, $k$ and $N$, it proves useful to introduce the two-variable generating function

$$
\mathcal{N}(w, z)=\sum_{N=1}^{\infty} \sum_{k=1}^{\infty}\left\langle N_{k}(N)\right\rangle w^{N-1} z^{k} .
$$

The governing equation for $\mathcal{N}(w, z)$ that is obtained from equation (30) is

$$
\left(2(1-w) \frac{\partial}{\partial w}+z(1-z) \frac{\partial}{\partial z}-2\right) \mathcal{N}=\frac{2 z}{(1-w)^{2}} .
$$

This is similar to equation (8) and can be solved accordingly. We introduce the rotated variables $x, y$ such that

$$
x+y=-\frac{1}{2} \ln (1-w) \quad x-y=\ln \frac{z}{1-z}
$$

to recast equation (32) into

$$
\left(\frac{\partial}{\partial x}-2\right) \mathcal{N}(x, y)=\frac{2 \mathrm{e}^{5 x+4 y}}{\mathrm{e}^{x}+\mathrm{e}^{y}} .
$$

The general solution is

$$
\mathcal{N}(x, y)=\mathrm{e}^{4 x+4 y}-2 \mathrm{e}^{3 x+5 y}+2 \mathrm{e}^{2 x+6 y} \ln \left(\mathrm{e}^{x}+\mathrm{e}^{y}\right)+\mathrm{e}^{2 x} G(y)
$$


and the function $G(y)$ is found from the initial condition $\mathcal{N}(w=0, z)=2 z$. When $w=0$, we have $x=-y=\frac{1}{2} \ln [z /(1-z)]$, and hence $\mathcal{N}(-y, y)=2 /\left(1+\mathrm{e}^{2 y}\right)$. Therefore

and finally

$$
G(y)=\frac{2 \mathrm{e}^{2 y}}{1+\mathrm{e}^{2 y}}-\mathrm{e}^{2 y}+2 \mathrm{e}^{4 y}-2 \mathrm{e}^{6 y} \ln \left(\mathrm{e}^{-y}+\mathrm{e}^{y}\right)
$$

$\mathcal{N}(x, y)=\mathrm{e}^{4 x+4 y}-2 \mathrm{e}^{3 x+5 y}-\mathrm{e}^{2 x+2 y}+2 \mathrm{e}^{2 x+4 y}+2 \frac{\mathrm{e}^{2 x+2 y}}{1+\mathrm{e}^{2 y}}+2 \mathrm{e}^{2 x+6 y} \ln \left(\frac{\mathrm{e}^{x+y}+\mathrm{e}^{2 y}}{1+\mathrm{e}^{2 y}}\right)$.

In terms of the original $w, z$ variables,

$$
\begin{gathered}
\mathcal{N}(w, z)=\frac{\left(3-2 z^{-1}\right)}{(1-w)^{2}}-\frac{1}{1-w}+\frac{2\left(z^{-1}-1\right)}{(1-w)^{3 / 2}}+\frac{2(1-w)^{-1 / 2}}{\left(z^{-1}-1\right)+(1-w)^{1 / 2}} \\
-\frac{2\left(z^{-1}-1\right)^{2}}{(1-w)^{2}} \ln \left[1-z+z(1-w)^{1 / 2}\right] .
\end{gathered}
$$

By expanding $\mathcal{N}(w, z)$, we can in principle determine all the $\left\langle N_{k}(N)\right\rangle$.

\subsection{Scaling function}

To extract the scaling function $F(\xi)$ from the generating function $\mathcal{N}(w, z)$ we use the same approach as in section 4 . The details are given in appendix $\mathrm{E}$ and the final result is

$$
F(\xi)=\operatorname{erfc}\left(\frac{\xi}{2}\right)+\frac{2 \xi+\xi^{3}}{\sqrt{4 \pi}} \mathrm{e}^{-\xi^{2} / 4}
$$

where $\operatorname{erfc}(x)$ is the complementary error function. A similar result for a related network model was found previously by Dorogovtsev et al [14]. Note that the exact form for $F(\xi)$ vanishes much more quickly than predicted by the continuum approach. When $k \gg \sqrt{N}$, the continuum approach gives

$$
\left\langle N_{k}(N)\right\rangle_{\text {cont. }} \rightarrow \frac{2}{\sqrt{N}} \mathrm{e}^{-k / \sqrt{N}}
$$

while the exact average degree distribution has a Gaussian large-degree tail

$$
\left\langle N_{k}(N)\right\rangle_{\text {exact }} \rightarrow \frac{2}{\sqrt{\pi N}} \mathrm{e}^{-k^{2} / 4 N} .
$$

The scaling function in equation (37) quantitatively accounts for the shoulder in the degree distribution. In contrast, while the scaling function from the continuum approach does exhibit a peak, it is both quantitatively and qualitatively inaccurate (figure 4).

\section{Higher moments and their fluctuation}

We now turn to higher moments of the degree distribution, as well as the fluctuation in these quantities between different realizations of the network. While the zeroth and first moments of the degree distribution are simply related to the total number of links for any network topology, the higher moments are not so simply characterized, but instead reflect the powerlaw tail of the degree distribution.

We first compare the moments of the average degree distribution to appreciate the difference between the continuum and exact descriptions. For the second moment, we use the identity

$$
\left.\sum_{k=1}^{\infty} k(k+1)\left\langle N_{k}\right\rangle \equiv\left(z^{2} \frac{\partial}{\partial z}\right)^{2} \mathcal{N}(N, z)\right|_{z=1}
$$




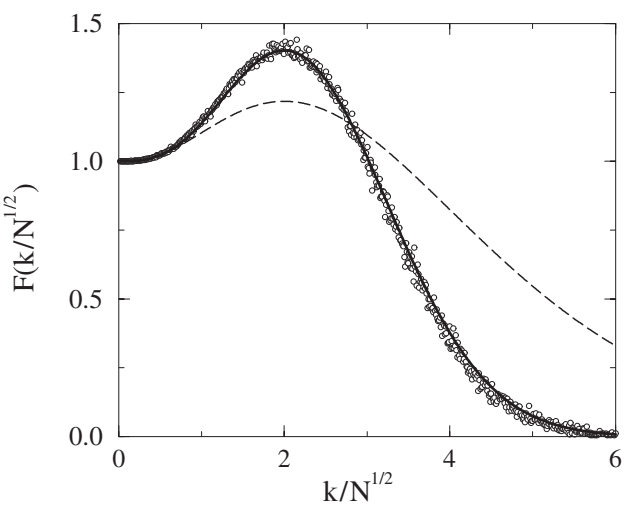

Figure 4. Comparison between the scaling function $F(\xi)$, with $\xi=k / N^{1 / 2}$, in the continuum approximation (equation (18), dashed curve) and in the discrete approach (equation (37), solid curve). The circles give the simulation data of $10^{6}$ realizations of a network with $N=10^{4}$ links for the dimer initial condition; these data coincide with the theoretical prediction.

Using $\mathcal{N}(N, z)$ from equation (11), together with the value of the first moment, we obtain, in the continuum approximation,

$$
\left\langle k^{2}\right\rangle_{\text {cont. }} \equiv \sum_{k=1}^{\infty} k^{2}\left\langle N_{k}\right\rangle_{\text {cont. }}=2 N \ln N+2 N \text {. }
$$

On the other hand, using the exact discrete expression (36) we find

$$
\left.\left(z^{2} \frac{\partial}{\partial z}\right)^{2} \mathcal{N}(w, z)\right|_{z=1}=\frac{4-2 \ln (1-w)}{(1-w)^{2}}
$$

which we then expand in a series in $w$ to yield, for the second moment,

$$
\left\langle k^{2}\right\rangle_{\text {exact }} \equiv \sum_{k=1}^{\infty} k^{2}\left\langle N_{k}\right\rangle_{\text {exact }}=2 N H_{N} .
$$

Here $H_{N}=\sum_{1 \leqslant j \leqslant N} j^{-1}$ is the harmonic number [16]. In the large $N$ limit, therefore,

$$
\left\langle k^{2}\right\rangle_{\text {exact }}=2 N \ln N+2 \gamma N+1-\frac{1}{6 N}+\cdots
$$

where $\gamma \cong 0.5772166$ is Euler's constant.

For higher moments, even the leading term given by the continuum approach is erroneous. For example,

$$
\left\langle k^{3}\right\rangle_{\text {cont. }}=24 N^{3 / 2}-6 N \ln N-22 N
$$

while the exact value is

$$
\left\langle k^{3}\right\rangle_{\text {exact }}=\frac{32}{\sqrt{\pi}} \frac{\Gamma\left(N+\frac{3}{2}\right)}{\Gamma(N)}-6 N H_{N}-16 N .
$$

More generally, the dependence of the moments on $N$ stems from the power-law tail of the degree distribution $\left\langle N_{k}\right\rangle \propto N / k^{3}$. From this asymptotic distribution, a suitably normalized set of measures for the mean degree

$$
\mathcal{M}_{n}=\left(\frac{\left\langle k^{n}\right\rangle}{\left\langle k^{0}\right\rangle}\right)^{1 / n}
$$




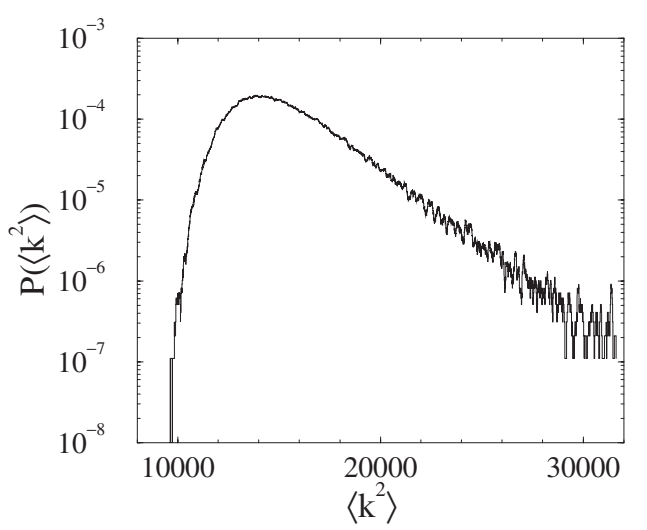

Figure 5. Distribution of $\left\langle k^{2}\right\rangle$ for $10^{5}$ realizations of a growing network with $N=10^{3}$ for attachment rate $A_{k}=k$ with the triangle initial condition. The raw data have been smoothed over a 100 -point range.

has the following $N$ dependence:

$$
\mathcal{M}_{n} \propto \begin{cases}\text { const. } & n<2 \\ \ln N & n=2 \\ N^{(n-2) / 2} & n>2 .\end{cases}
$$

In a related vein, we also study the fluctuations in these moments between different realizations of the network growth. That is, we record the value of $\left\langle k^{2}\right\rangle$ for each realization of the network to obtain the underlying distribution $P\left(\left\langle k^{2}\right\rangle\right)$. A typical result is shown in figure 5 . Note that the distribution of $\left\langle k^{2}\right\rangle$ is relatively broad with an exponential tail. The distributions of higher moments are even broader, with each being dominated by the realizations with the largest value of the corresponding moment.

\section{Concluding remarks}

We studied the role of finiteness on the degree distributions of growing networks with a node attachment rate of the form $A_{k}=k+\lambda$. For finite networks, fluctuations are no longer negligible and a stochastic approach is needed to analyse these properties. We found the average degree distribution within an approximate continuum formulation and by an exact discrete approach. The continuum approach has the advantage of being much simpler than the discrete formulation, but does not provide a quantitatively accurate description of the large- $k$ tail of the degree distribution.

We also argued that the degree distribution $N_{k}(N)$, when considered as the random variable in $k$, exhibits self-averaging, i.e., the relative fluctuations in $N_{k}(N)$ diminish as $N \rightarrow \infty$. Moreover, the variance $\sigma_{k}^{2}=\left\langle N_{k}^{2}\right\rangle-\left\langle N_{k}\right\rangle^{2}$ varies linearly with $N$, and the probability distribution $P\left(N_{k}, N\right)$ approaches a Gaussian. To support these assertions, we computed $\sigma_{k}^{2}$ for $k=1,2$. These partial results support our general hypothesis that fluctuations in $N_{k}(N)$ are Gaussian. Perhaps, the Van Kampen $\Omega$-expansion [17] would prove to be a more appropriate analysis tool to undertake a systematic study of fluctuations in growing networks.

Of course, the random variables $N_{k}(N)$ should be Gaussian only for sufficiently small $k$, namely, as long as $\left\langle N_{k}\right\rangle \gg 1$, or equivalently, $k \ll N^{1 /(3+\lambda)}$. On the other hand, fluctuations become large and non-Gaussian when $k \propto N^{1 /(3+\lambda)}$. Determining the fluctuations in this degree 
range seems to be difficult, as one must study the master equation for the joint probability distribution.

In this work, we limited ourselves to the degree distribution; this is perhaps the most important and also the most easily analysable local structural characteristic of a network. However, recent investigations of growing networks have increasingly focused on global characteristics, such as the size distribution of connected components, see e.g., [18-21]. The methods described in this paper should be applicable to probing fluctuations of the component size distribution and other global network characteristics. This direction seems especially exciting since the simplest growing network models that allow for a multiplicity of clusters exhibit a very unusual infinite-order percolation transition [18-21]. Thus, one might anticipate interesting giant fluctuations near the percolation transition of these models.

\section{Acknowledgment}

We are grateful to NSF grant DMR9978902 for partial financial support of this research.

\section{Appendix A. The average degree distribution in the continuum formulation}

Within the continuum framework, the average degree distribution is described by equations (4). Successively solving these equations by elementary methods, we obtain $\left\langle N_{k}(N)\right\rangle$. For $k=1,2,3$ and 4 we obtain

$$
\begin{aligned}
& \left\langle N_{1}(N)\right\rangle=\frac{2}{3} N+\frac{4}{3} \frac{1}{N^{1 / 2}} \\
& \left\langle N_{2}(N)\right\rangle=\frac{1}{6} N+\frac{4}{3} \frac{1}{N^{1 / 2}}-\frac{3}{2} \frac{1}{N} \\
& \left\langle N_{3}(N)\right\rangle=\frac{1}{15} N+\frac{4}{3} \frac{1}{N^{1 / 2}}-3 \frac{1}{N}+\frac{8}{5} \frac{1}{N^{3 / 2}} \\
& \left\langle N_{4}(N)\right\rangle=\frac{1}{30} N+\frac{4}{3} \frac{1}{N^{1 / 2}}-\frac{9}{2} \frac{1}{N}+\frac{24}{5} \frac{1}{N^{3 / 2}}-\frac{5}{3} \frac{1}{N^{2}} .
\end{aligned}
$$

\section{Appendix B. Generating function for $\left\langle N_{1}^{2}(N)\right\rangle$}

To determine $\left\langle N_{1}^{2}(N)\right\rangle$, we introduce the generating function $\mathcal{Y}_{1}(w)=\sum_{N \geqslant 1}\left\langle N_{1}^{2}(N)\right\rangle w^{N-1}$. This converts the recursion relation equation (25) into the differential equation for the generating function

$$
(1-w) \frac{\mathrm{d} \mathcal{Y}_{1}}{\mathrm{~d} w}=\frac{1}{(1-w)^{2}}+\frac{3}{2} \mathcal{X}_{1}+2 w \frac{\mathrm{d} \mathcal{X}_{1}}{\mathrm{~d} w}
$$

with $\mathcal{X}_{1}(w)$ given by equation (23). Solving (B1) subject to the initial condition $\mathcal{Y}_{1}(0)=4$ we obtain

$\mathcal{Y}_{1}(w)=\frac{8}{9} \frac{1}{(1-w)^{3}}-\frac{1}{3} \frac{1}{(1-w)^{2}}+\frac{8}{9} \frac{1}{(1-w)^{3 / 2}}-\frac{4}{3} \frac{1}{(1-w)^{1 / 2}}+\frac{35}{9}$.

Expanding this generating function in a Taylor series then yields the result for $\left\langle N_{1}^{2}(N)\right\rangle$ quoted in equation (26).

\section{Appendix C. Generating function for first moment}

Here we solve the recursion formula (30) for $\left\langle N_{k}(N)\right\rangle$. We first introduce the generating function $\mathcal{X}_{k}(w)=\sum_{N=1}^{\infty}\left\langle N_{k}(N)\right\rangle w^{N-1}$ to eliminate the variable $N$ and convert equation (30) 
into a differential equation that relates $\mathcal{X}_{k}$ and $\mathcal{X}_{k-1}$. This equation is further simplified by making the transformation

$$
\mathcal{X}_{k}(w)=(1-w)^{\frac{k}{2}-1} \mathcal{U}_{k}(u) \quad u=\frac{1}{\sqrt{1-w}}-1 .
$$

The resulting equation is

$$
\frac{\mathrm{d} \mathcal{U}_{k}}{\mathrm{~d} u}=(k-1) \mathcal{U}_{k-1} \quad k \geqslant 2
$$

Rewriting our previous solution (23) as

$$
\mathcal{U}_{1}(u)=\frac{2}{3} u^{3}+2 u^{2}+2 u+2
$$

one can solve equations (C2) subject to the initial condition $\mathcal{U}_{k}(u=0)=0$ for $k \geqslant 2$. The final result is

$$
\mathcal{U}_{k}(u)=\frac{4 u^{k+2}}{k(k+1)(k+2)}+\frac{4 u^{k+1}}{k(k+1)}+\frac{2 u^{k}}{k}+2 u^{k-1} .
$$

Using the binomial formula, we transform $\mathcal{X}_{k}(z)$ into the series

$$
\begin{aligned}
& \mathcal{X}_{k}(w)=\frac{4}{k(k+1)(k+2)} \frac{1}{(1-w)^{2}}+\frac{4}{3} \frac{1}{(1-w)^{1 / 2}} \\
& +2 \sum_{a=1}^{k-1}(-1)^{a} \frac{a+2}{a+3}\left(\begin{array}{c}
k-1 \\
a
\end{array}\right)(1-w)^{(a-1) / 2} .
\end{aligned}
$$

Expanding $\mathcal{X}_{k}(w)$ in a Taylor series in $w$ we obtain $\left\langle N_{k}(N)\right\rangle$. The analytic expressions for $\left\langle N_{k}(N)\right\rangle$ with $k \leqslant 5$ are obtained by expanding $\mathcal{X}_{k}(w)$ in a Taylor series. This gives

$$
\begin{aligned}
&\left\langle N_{1}(N)\right\rangle= \frac{2}{3} N+\frac{4}{3 \sqrt{\pi}} \frac{\Gamma\left(N-\frac{1}{2}\right)}{\Gamma(N)} \\
&\left\langle N_{2}(N)\right\rangle= \frac{1}{6} N+\frac{4}{3 \sqrt{\pi}} \frac{\Gamma\left(N-\frac{1}{2}\right)}{\Gamma(N)}-\frac{3}{2} \delta_{N, 1} \\
&\left\langle N_{3}(N)\right\rangle= \frac{1}{15} N+\frac{4}{3 \sqrt{\pi}} \frac{\Gamma\left(N-\frac{1}{2}\right)}{\Gamma(N)}-3 \delta_{N, 1}-\frac{4}{5 \sqrt{\pi}} \frac{\Gamma\left(N-\frac{3}{2}\right)}{\Gamma(N)} \\
&\left\langle N_{4}(N)\right\rangle= \frac{1}{30} N+\frac{4}{3 \sqrt{\pi}} \frac{\Gamma\left(N-\frac{1}{2}\right)}{\Gamma(N)}-\frac{9}{2} \delta_{N, 1}-\frac{12}{5 \sqrt{\pi}} \frac{\Gamma\left(N-\frac{3}{2}\right)}{\Gamma(N)}-\frac{5}{3} \delta_{N, 1}+\frac{5}{3} \delta_{N, 2} \\
&\left\langle N_{5}(N)\right\rangle= \frac{2}{105} N+\frac{4}{3 \sqrt{\pi}} \frac{\Gamma\left(N-\frac{1}{2}\right)}{\Gamma(N)}-6 \delta_{N, 1}-\frac{24}{5 \sqrt{\pi}} \frac{\Gamma\left(N-\frac{3}{2}\right)}{\Gamma(N)} \\
& \quad-\frac{20}{3} \delta_{N, 1}+\frac{20}{3} \delta_{N, 2}+\frac{9}{7 \sqrt{\pi}} \frac{\Gamma\left(N-\frac{5}{2}\right)}{\Gamma(N)} .
\end{aligned}
$$

Generally, there are slightly different formulae for even

$$
\left\langle N_{2 k}(N)\right\rangle=n_{2 k} N+\sum_{j=1}^{k} A_{k j} \delta_{N j}+\sum_{i=0}^{k-1} \frac{4(i+1)}{2 i+3}\left(\begin{array}{c}
2 k-1 \\
2 i
\end{array}\right) \frac{\Gamma\left(N-\frac{1}{2}-i\right)}{\Gamma\left(\frac{1}{2}-i\right) \Gamma(N)}
$$

and odd

$\left\langle N_{2 k+1}(N)\right\rangle=n_{2 k+1} N+\sum_{j=1}^{k} B_{k j} \delta_{N j}+\sum_{i=0}^{k} \frac{4(i+1)}{2 i+3}\left(\begin{array}{c}2 k \\ 2 i\end{array}\right) \frac{\Gamma\left(N-\frac{1}{2}-i\right)}{\Gamma\left(\frac{1}{2}-i\right) \Gamma(N)}$

indices. Here the $n_{k}$ are given by equations (2) and explicit expressions for the coefficients $A_{k j}$ and $B_{k j}$ could be found by expanding the polynomials in the generating functions $\mathcal{X}_{2 k}(w)$ and $\mathcal{X}_{2 k+1}(w)$ 


\section{Appendix D. Higher moments}

Starting from equation (29), a straightforward computation yields

$$
\left\langle N_{k}^{2}\right\rangle=\left(1-\frac{k}{N}\right)\left\langle N_{k}^{2}\right\rangle+\frac{k-1}{N}\left\langle N_{k-1} N_{k}\right\rangle+\left\langle\frac{(k-1) N_{k-1}+k N_{k}}{2 N}\right\rangle
$$

where the correlation function on the left-hand side is a function of $N+1$ and those on the right-hand side are functions of $N$. Obviously, $\left\langle N_{k}^{2}\right\rangle$ is coupled with $\left\langle N_{k-1} N_{k}\right\rangle$. The recursion relation for this correlation function reads (for $k \geqslant 3$ )

$$
\left\langle N_{k-1} N_{k}\right\rangle=\left(1-\frac{2 k-1}{2 N}\right)\left\langle N_{k-1} N_{k}\right\rangle+\frac{k-1}{2 N}\left\langle N_{k-1}^{2}\right\rangle+\frac{k-2}{2 N}\left\langle N_{k-2} N_{k}\right\rangle-\frac{k-1}{2 N}\left\langle N_{k-1}\right\rangle .
$$

Fortunately, no higher-order correlation functions appear, and additionally the total index decreases, i.e., $\left\langle N_{k}^{2}\right\rangle$, whose total index is $2 k$, involves the correlation function $\left\langle N_{k-1} N_{k}\right\rangle$, whose total index is $2 k-1$. One therefore can determine all correlation functions by starting from the smallest total index and then working up to larger indices. For example, the first non-trivial correlation function $\left\langle N_{1} N_{2}\right\rangle$ whose total index equals three satisfies an equation slightly different from the general form of equation (D2), namely,

$$
\left\langle N_{1} N_{2}\right\rangle=\left(1-\frac{3}{2 N}\right)\left\langle N_{1} N_{2}\right\rangle+\frac{1}{2 N}\left\langle N_{1}^{2}\right\rangle+\left(1-\frac{1}{N}\right)\left\langle N_{2}\right\rangle
$$

Note here that we already know $\left\langle N_{1}^{2}\right\rangle$.

We can solve for $\left\langle N_{1} N_{2}\right\rangle$ using the generating function technique. We define the generating function $\mathcal{Z}_{1}(w)=\sum_{N \geqslant 1}\left\langle N_{1}(N) N_{2}(N)\right\rangle w^{N-1}$ which satisfies the differential equation

$$
2(1-w) \frac{\mathrm{d} \mathcal{Z}_{1}}{\mathrm{~d} w}=-\mathcal{Z}_{1}+\mathcal{Y}_{1}+2 w \frac{\mathrm{d} \mathcal{X}_{2}}{\mathrm{~d} w}
$$

with solution

$\mathcal{Z}_{1}(w)=\frac{2}{9} \frac{1}{(1-w)^{3}}-\frac{1}{5} \frac{1}{(1-w)^{2}}+\frac{5}{9} \frac{1}{(1-w)^{3 / 2}}-\frac{4}{3} \frac{1}{(1-w)^{1 / 2}}-\frac{47}{15}(1-w)^{1 / 2}+\frac{35}{9}$.

Expanding $\mathcal{Z}_{1}(w)$ in a power series in $w$ we obtain

$$
\begin{gathered}
\left\langle N_{1} N_{2}\right\rangle=\frac{1}{9} N(N+1)-\frac{1}{5} N+\frac{10}{9 \sqrt{\pi}} \frac{\Gamma\left(N+\frac{1}{2}\right)}{\Gamma(N)}-\frac{4}{3 \sqrt{\pi}} \frac{\Gamma\left(N-\frac{1}{2}\right)}{\Gamma(N)} \\
+\frac{47}{30 \sqrt{\pi}} \frac{\Gamma\left(N-\frac{3}{2}\right)}{\Gamma(N)}+\frac{35}{9} \delta_{N, 1} .
\end{gathered}
$$

Asymptotically, $\left\langle N_{1} N_{2}\right\rangle \rightarrow\left\langle N_{1}\right\rangle\left\langle N_{2}\right\rangle$ as expected.

There are two correlation functions, $\left\langle N_{2}^{2}\right\rangle$ and $\left\langle N_{1} N_{3}\right\rangle$, whose total index equals four. The former satisfies equation (D1) with $k=2$, i.e.,

$$
\left\langle N_{2}^{2}\right\rangle=\left(1-\frac{2}{N}\right)\left\langle N_{2}^{2}\right\rangle+\left\langle\frac{N_{1}+2 N_{2}+2 N_{1} N_{2}}{2 N}\right\rangle
$$

from which we determine the generating function

$$
\begin{aligned}
\mathcal{Y}_{2}(w)=\frac{1}{18} & \frac{1}{(1-w)^{3}}+\frac{1}{10} \frac{1}{(1-w)^{2}}+\frac{2}{9} \frac{1}{(1-w)^{3 / 2}} \\
& +\frac{4}{9} \frac{1}{(1-w)^{1 / 2}}-\frac{94}{15}(1-w)^{1 / 2}+\frac{49}{9}-\frac{55}{18} w .
\end{aligned}
$$


Expanding $\mathcal{Y}_{2}(w)$ we obtain

$$
\begin{gathered}
\left\langle N_{2}^{2}(N)\right\rangle=\frac{1}{36} N(N+1)+\frac{1}{10} N+\frac{4}{9 \sqrt{\pi}} \frac{\Gamma\left(N+\frac{1}{2}\right)}{\Gamma(N)}+\frac{4}{9 \sqrt{\pi}} \frac{\Gamma\left(N-\frac{1}{2}\right)}{\Gamma(N)} \\
+\frac{47}{15 \sqrt{\pi}} \frac{\Gamma\left(N-\frac{3}{2}\right)}{\Gamma(N)}+\frac{49}{9} \delta_{N, 1}-\frac{55}{18} \delta_{N, 2} .
\end{gathered}
$$

In the large $N$ limit, we find that variance grows linearly with $N$ according to $\sigma_{2}^{2} \sim \frac{23}{180} N$. It appears that

$$
\sigma_{k}^{2} \rightarrow \mu_{k} N \quad \text { as } \quad N \rightarrow \infty
$$

for all $k$, although we solved only the cases $k=1$ and 2 , where $\mu_{1}=\frac{1}{9}$ and $\mu_{2}=\frac{23}{180}$.

\section{Appendix E. Scaling function in the discrete approach}

To extract the scaling function from the generating function $\mathcal{N}(w, z)$ we adapt the technique employed in section 4 for discrete variables. We first write

$$
z^{-1}=1+s \sqrt{1-w}
$$

and keep $s$ finite while taking the $w \rightarrow 1$ limit. We again consider the modified generating function

$$
\left(z^{2} \frac{\partial}{\partial z}\right)^{3} \mathcal{N}=\sum_{N=1}^{\infty} \sum_{k=1}^{\infty} 4 N F\left(\frac{k}{\sqrt{N}}\right) w^{N-1} z^{k+3} .
$$

On the right-hand side of this equation we have already replaced $(k+2)(k+1) k\left\langle N_{k}(N)\right\rangle$ by $4 N F(k / \sqrt{N})$ as implied by equations (2) and (3).

Substituting the exact expression (36) for the generating function into the left-hand side of equation (E2) and keeping only the dominant contribution gives

$$
4(1-w)^{-5 / 2} J(s)
$$

with $J(s)$ given by equation (15). To simplify the right-hand side of equation (E2) we substitute equation (E1) and replace the sums by integrals. The dominant contribution in the $w \rightarrow 1$ limit is

$$
4(1-w)^{-5 / 2} \int_{0}^{\infty} \mathrm{d} \xi \mathrm{e}^{-\xi s} \int_{0}^{\infty} \mathrm{d} \eta \eta F\left(\xi \eta^{-1 / 2}\right) \mathrm{e}^{-\eta}
$$

where $\xi=k \sqrt{1-w}$ and $\eta=N(1-w)$. Therefore the double integral in equation (E4) is equal to $J(s)$. The double integral can be interpreted as the Laplace transform $\hat{\Phi}(s)=$ $\int_{0}^{\infty} \mathrm{d} \xi \exp (-s \xi) \Phi(\xi)$ of the function

$$
\Phi(\xi)=\int_{0}^{\infty} \mathrm{d} \eta \eta F\left(\xi \eta^{-1 / 2}\right) \mathrm{e}^{-\eta}
$$

We already know how to solve $\hat{\Phi}(s)=J(s)$, so

$$
\Phi(\xi)=(1+\xi)\left(1+\frac{\xi^{2}}{2}\right) \mathrm{e}^{-\xi}
$$

To determine $F(\xi)$, we must solve the integral equation (E6) with $\Phi(\xi)$ given by equation (E5). To solve this integral equation, note that $\Phi(\xi)$ is almost a Laplace transform of function $F$. Indeed, if instead of $\eta$ and $F\left(\xi \eta^{-1 / 2}\right)$ we use $\zeta$ and $G(\zeta)$ defined according to

$$
\zeta=\frac{\eta}{\xi^{2}} \quad G(\zeta)=\zeta F\left(\zeta^{-1 / 2}\right)
$$


then we obtain $\Phi(\xi)=p^{2} \hat{G}(p)$, with $p=\xi^{2}$ being the Laplace variable and $\hat{G}(p)=$ $\int_{0}^{\infty} \mathrm{d} \zeta G(\zeta) \exp (-p \zeta)$. Re-writing the integral equation (E6) in terms of $p$ gives

$$
\hat{G}(p)=\left(p^{-2}+p^{-3 / 2}+\frac{1}{2} p^{-1}+\frac{1}{2} p^{-1 / 2}\right) \exp (-\sqrt{p}) .
$$

Inverting this Laplace transform yields [22]

$$
G(\zeta)=\zeta \operatorname{erfc}\left(\frac{1}{\sqrt{4 \zeta}}\right)+\frac{2 \zeta+1}{\sqrt{4 \pi \zeta}} \mathrm{e}^{-1 / 4 \zeta}
$$

where $\operatorname{erfc}(x)=\frac{2}{\sqrt{\pi}} \int_{x}^{\infty} \mathrm{d} t \exp \left(-t^{2}\right)$ is the complementary error function. Since $F(\xi)=$ $\xi^{2} G\left(\xi^{-2}\right)$, see equation (E7), we arrive at the scaled average degree distribution quoted in equation (37).

\section{References}

[1] Broder A et al 2000 Comput. Netw. 33309

[2] Willinger W et al 2002 Proc. Natl. Acad. Sci. 992573

[3] Krapivsky P L and Redner S 2002 Comput. Netw. 39262

[4] Huberman B A and Adamic L A 1999 Nature 401131

[5] Moreira A A, Andrade J S and Amaral L A N 2002 Preprint cond-mat/0205411

[6] Krapivsky P L and Redner S 2002 Preprint cond-mat/0207370

[7] Simon H A 1955 Biometrica 42425

Simon H A 1960 Inf. Control 380

[8] Barabási A-L and Albert R 1999 Science 286509

[9] Krapivsky P L and Redner S 2001 Phys. Rev. E 63066123

[10] Krapivsky P L, Redner S and Leyvraz F 2000 Phys. Rev. Lett. 854629

[11] Dorogovtsev S N, Mendes J F F and Samukhin A N 2000 Phys. Rev. Lett. 854633

[12] Zanette D H and Manrubia S C 2001 Physica A 2951

[13] Kullmann L and Kertész J 2001 Phys. Rev. E 63051112

[14] Dorogovtsev S N, Mendes J F F and Samukhin A N 2001 Phys. Rev. E 63062101

[15] Burda Z, Correia J D and Krzywicki A 2001 Phys. Rev. E 64046118

[16] Graham R L, Knuth D E and Patashnik O 1989 Concrete Mathematics: A Foundation for Computer Science (Reading, MA: Addison-Wesley)

[17] Van Kampen N G 1981 Stochastic Processes in Physics and Chemistry (Amsterdam: North-Holland)

[18] Callaway D S et al 2001 Phys. Rev. E 64041902

[19] Dorogovtsev S N, Mendes J F F and Samukhin A N 2001 Phys. Rev. E 64066110

[20] Kim J, Krapivsky P L, Kahng B and Redner S 2002 Preprint cond-mat/0203167

[21] Bauer M and Bernard D 2002 Preprint cond-mat/0203232

[22] Abramowitz M and Stegun I A 1970 Handbook of Mathematical Functions (New York: Dover) 